\title{
Stadt von rechts?
}

\author{
Eine Einleitung
}

\author{
Tobias Bernet, Peter Bescherer, \\ Kristine Beurskens, Robert Feustel, \\ Boris Michel
}

Trump, Bolsonaro und Salvini, Höcke, Seehofer und Gauland. Der Wind weht von rechts: Europa schottet sich ab, lässt Menschen sehenden Auges sterben und kriminalisiert die Seenotrettung. Die innere Sicherheit dominiert die Schlagzeilen und immer neue Phantasmen des Fremden werden über den Boulevard gejagt. Wie es so weit kommen konnte, was den Rechtsruck ausgelöst und was ihn begünstigt hat, ist heftig umstritten. Zwei Pole lassen sich ausmachen, zwischen denen sich die Debatte abspielt: Einerseits heißt es, es handele sich vor allem um eine Reaktion auf steigende soziale Ungleichheiten, die vor dem Hintergrund der bröckelnden Hegemonie des Neoliberalismus von rechten Akteuren genutzt werde. Rassismus und autoritäre Einstellungen seien vorrangig eine Antwort auf soziale Benachteiligung, Abstiegsängste und politische Enttäuschung und müssten durch eine andere Verteilung eingedämmt werden. Gegen diese These wird andererseits argumentiert, dass der gesellschaftlich tief verankerte und lange tradierte Rassismus eine eigenständige Herrschaftsform sei, die sich nicht sozial kleinarbeiten, sondern nur (frontal, mit allen Mitteln) bekämpfen lasse. In dieser Debatte werden häufig Argumente und Studien aus der Forschung zum Wandel der Arbeitswelt, zur Zunahme sozialer Ungleichheit und zu Verzerrungen in der politischen Repräsentation herangezogen (etwa Dörre et al. 2018), gegen die wiederum Erkenntnisse zu Rassismus oder feministische Theorie vorgebracht werden (etwa Dowling et al. 2017). Die politische und wissenschaftliche Diskussion zu den Gründen der aktuellen politischen Lage ist in vollem Gange.[1]

Welche Rolle raumbezogene Differenzierungen für die Ausbildung und Stabilisierung rechter Orientierungen spielen, wird dagegen weniger intensiv und eher klischeehaft diskutiert. Häufig nehmen die Kommentator_innen dabei eine Gegenüberstellung von Ost und West oder von kosmopolitischen Städten und reaktionären Dörfern vor. Diese Diskussionen können auf eine lange Tradition zurückgreifen, die bis in die Mitte des 19. Jahrhunderts zurückreicht. Einerseits wurde konservative bis rassistische Kulturkritik vielfach großstadtfeindlich formuliert (vgl. u. a. Riehl 1853, Spengler 1918, 1922). Andererseits galt die Stadt vielen liberalen und linken Beobachter_innen als die räumliche Manifestation oder zumindest als Möglichkeitsraum 
von Fortschritt und sie sahen in der „Entbarbarisierung auf dem platten Land" (Adorno 1977 [1967]: 680) einen wesentlichen Auftrag postfaschistischer Erziehung. Der sub\urban-Schwerpunkt „Stadt von rechts?" stellt sich diesem Thema und versucht, ein wenig Klarheit in die Auseinandersetzung zu bringen. Sind tatsächlich die Städte Orte der Hoffnung und Toleranz und die Dörfer solche der Stagnation und Borniertheit? Haben wir es gar mit einer „Rache der Dörfer“ zu tun, wie es Wolfgang Kaschuba (2016) pointiert formuliert? Die Dinge sind komplizierter, wie dieser Schwerpunkt ausleuchten soll.

Die drei Gastherausgeber des Schwerpunkts sind auf unterschiedliche Weise schon länger mit dem Thema beschäftigt, wissenschaftlich und politisch. Im Forschungsprojekt PODESTA (Populismus und Demokratie in der Stadt)[2], in dem Peter Bescherer und Robert Feustel arbeiten, wird anhand von Leipzig und Stuttgart untersucht, ob und inwiefern städtische Problemlagen (etwa die Wohnraumversorgung oder die häufig unzulängliche Beteiligung an städtebaulichen Projekten) ein Gelegenheitsfenster für rechte Parteien, Bewegungen und Strömungen schaffen und wie städtische soziale, linke und radikaldemokratische Bewegungen darauf reagieren. In den Projektverbund sind Praxispartner_innen aus zivilgesellschaftlichen Initiativen eingebunden, in Leipzig das Netzwerk „Stadt für alle“, in dem Tobias Bernet mitwirkt, der sich wissenschaftlich mit der Wohnungsfrage beschäftigt. Zum Hintergrund des Themenheftes gehört auch, dass die drei eine lebensweltliche Erfahrung teilen: Sie kommen alle aus dem Kontext sozialer Bewegungen und leben in sogenannten Szenevierteln in Leipzig - in einer Stadt also, die in Sachsen liegt und im Selbstverständnis vieler Leipziger_innen zugleich ganz anders ist als der Rest des Bundeslandes. Der Drang, das Verhältnis zwischen dem angeblich roten Leipzig, wie die AfD die Stadt gern beschreibt, und ihrem Hinterland auch aus dieser Perspektive genauer in den Blick zu nehmen, ist als Motiv für die Arbeit am Themenschwerpunkt wichtig gewesen. Schließlich haben sich auch in Leipzig, trotz der erfolgreichen Proteste gegen den Leipziger Pegida-Ableger namens Legida, die Sagbarkeitsgrenzen nach rechts verschoben. Beispielhaft ist etwa der örtliche Sicherheitsdiskurs. Debatten um ein Bettelverbot, die durch marginalisierte Gruppen angeblich beeinträchtigte Aufenthaltsqualität am Hauptbahnhof, sogenannte Komplexkontrollen durch den zur Stadtpolizei aufgewerteten Ordnungsdienst und die symbolträchtige Einrichtung einer Waffenverbotszone zeigen, wie es aussieht, wenn sich Kommunalpolitik und -verwaltung von rechts getrieben fühlen.

Für die Redaktion von sub \urban liegt die Auseinandersetzung mit Raum, räumlicher sozialer Praxis und Politik beziehungsweise politischen Einstellungen zweifellos nahe. In der raumbezogenen Forschung hat die Debatte um rechte Bewegungen und Diskurse in den vergangenen Jahren stark zugenommen: Die Erfolge der AfD, die vielfach bestimmten Orten oder Regionen zugeschrieben werden, haben zu einer Wiederbelebung von Wahlgeographien geführt (Spier 2016, D’Antonio 2017, Oßenbrügge 2018). Rechte Aussagen werden auf Raumbezüge und Raumsemantiken hin untersucht, etwa wie sie sich in Diskursen der Abgrenzung von „Fremden“ (Eckardt 2018) oder der Klage über die Vernachlässigung durch Staat und Politik manifestieren (Hillje 2018). Auch die raumbezogenen Praktiken rechter Akteure werden untersucht, etwa die versuchte Aneignung urbaner 
öffentlicher Räume (Keller/Berger 2017). Besonders die Annahme schroffer Stadt-Land-Differenzen versucht die kritische (Stadt-)forschung mit gezielten Analysen der Bedeutungen von Urbanität und raumbezogenen Abhängigkeiten im Kontext neuer rechter Bewegungen aufzubrechen (vgl. Förtner et al. in diesem Heft). Tatsächlich lassen sich andere Differenzen erkennen, etwa jene zwischen Zentrum und Peripherie oder zwischen Urbanität und Ruralität, die nicht mit Stadt und Land deckungsgleich sein müssen. Nicht zuletzt wird der Gebrauch digitaler Medien daraufhin untersucht, wie er verschiedene Sozialräume beeinflusst und ob sich Übertragungseffekte aus den virtuellen in die physischen Räume beobachten lassen (vgl. Becker et al. 2018).

Stadt und Land sind nicht die einzigen politisch unsauber sortierten Schubladen. Schon der Begriff rechts ist klärungsbedürftig. Mitunter ist von den neuen (oder der Neuen, wir kommen darauf zurück) und der alten Rechten die Rede. Auch die Bezeichnungen konservativ, reaktionär, oder rechtspopulistisch sowie Neuschöpfungen wie ,autoritärer Nationalradikalismus“ (Heitmeyer 2018) zeugen von den Schwierigkeiten, diese Positionen auf den Begriff zu bringen. Die Beiträge des Themenschwerpunkts sprechen zumeist schlicht von rechten Parteien, Ideen et cetera, manchmal von der extremen Rechten. Wir meinen, dass eine Distanz zu Debatten angebracht ist, die anhand begrifflicher Ausdifferenzierungen zwischen demokratisch noch akzeptablen und eindeutig ungenießbaren politischen Positionen allzu bequeme Trennschärfen suggerieren.

Die terminologischen Probleme sind also vielfältig. Das betrifft zunächst den Extremismusbegriff beziehungsweise die Extremismusdoktrin, die von einer der Demokratiefeindlichkeit und Gewaltaffinität stets unverdächtigen, zum normativen Maß erhobenen Mitte ausgeht und beständig links und rechts auf eine Stufe stellt (vgl. FKR 2011). Ob jemand in Chemnitz den Hitlergruß zeigt und Menschen jagt oder im Hambacher Forst einen Baum besetzt, ist aus Sicht dieser formalistischen Logik gleichermaßen ,extremistisch (vgl. Stärck 2018). Extremismustheoretisch sind Rassismus, Antisemitismus und Sexismus genauso abweichend wie die Kritik daran. Dies verschleiert nicht zuletzt die starken Verflechtungen zwischen antidemokratisch-faschistischen Kräften und einem dem legitimen demokratischen Spektrum zugerechneten, jedoch mindestens latent rassistischen Konservativismus, wie ihn Exponent_innen der CSU oder der US-Republikaner_innen (auch schon vor Donald Trump) pflegen. Kurz: Die Extremismusdoktrin ist unbrauchbar, weil sie die Demokratie entpolitisiert, zwingend nötige Differenzierungen auslässt und unkritisch den Ist- zum Soll-Zustand umdeutet.

Auch das Etikett rechtspopulistisch ist angesichts offensichtlicher völkisch-autoritärer Konturen der AfD problematisch. Es klingt danach als verweise es auf eine eher harmlosere Spielart von rechtem Denken und verharmlost damit schroffe, völkische Ausgrenzungsphantasien und den deutlich erkennbaren Hang zum Autoritären, konkret zur plebiszitären Führerdemokratie, wie sie bereits Max Weber (1976 [1922]: 156f.) beschrieben hat. Demokratie ist dieser Vorstellung nach die unmittelbare Exekution des Volkswillens. Diese Form der Herrschaft kennt weder kompromissorientierte Aushandlungsprozesse noch die verfassungsmäßig verankerten Grundrechte und die demokratisch unerlässlichen Minderheitenrechte. 
Mit dem Grundwort Populismus, als dessen Spielart der Rechtspopulismus firmiert, dürfen wir es uns aber nicht zu einfach machen. Der Bezug auf den populus als Subjekt der Demokratie ist ja nicht grundsätzlich verkehrt (Rancière 2010) und ein wohlfeiler „liberaler Antipopulismus“ kündet weniger von Gesellschaftskritik denn von einer „zweiten Phase der Postpolitik“ (Marchart 2017: 16). Sofern politisches Handeln mehr ist als Technokratie, so das Argument von Ernesto Laclau (2005), ist Politik immer auf irgendeine Wiese populistisch, weil sie auf die Ausweitung von Äquivalenzketten zu einem Volk zielt. Die Frage ist allerdings, ob Volk ein sozial inklusiver Begriff ist - wie im Fall von, the people - oder ob er völkisch und rassifizierend, also ausgrenzend verwendet wird. Die Diskussionen zu Begriff und Gegenstand des Populismus sind weitverzweigt (vgl. Feustel/Bescherer 2018). Allerdings ist deutlich erkennbar, dass besonders in der deutschen Tradition die Kategorie ,Volk' seit dem letzten Drittel des 19. Jahrhunderts vom rassenideologischen Denken geprägt und damit immer problematisch ist.

Trotz der komplexen begrifflichen Gehalte ist jedoch erkennbar, dass das Wort ,Populismus', angewendet auf gegenwärtige rechte Bewegungen und Diskurse, den rassistischen, ausschließenden und autoritären Kern der Rechten entweder nicht hinreichend markiert oder sogar verdeckt. Dennoch kommen wir nicht ganz um den Begriff herum. Einerseits, weil es eine etablierte politische und mediale Praxis und damit eine Art unausgesprochenen Konsens gibt, u. a. die AfD und ihr Auftreten so zu bezeichnen. Und andererseits, weil sich derzeit ein neuer und in gewisser Weise tatsächlich populistischer Politikstil herausbildet, der die AfD und einige politische Akteure anderer Parteien (etwa Horst Seehofer, Boris Palmer oder Thilo Sarrazin) von älteren Parteien wie der NPD unterscheidet. Bei dieser Unterscheidung geht es weniger um Inhalte und Ziele, sondern um Taktiken und Stilmittel (vgl. Olschanski 2017, siehe auch Rancière 2017, Bonikowski et al 2019, Wirz 2018). Wir haben es also mit einem Dilemma zu tun: Als analytischer Begriff verschleiert und verharmlost ,Rechtspopulismus' einerseits die Dimension des so bezeichneten gesellschaftlichen Phänomens, andererseits ist der Ausdruck gängig und markiert einen demagogischen Politikstil eigener Prägung.

Für ähnliche Unklarheiten sorgt das ebenfalls häufig verwendete Attribut konservativ, das rechte Akteure gern für sich in Anspruch nehmen. Stilbildend für diese Diskussion ist Götz Kubitschek, Herausgeber der Zeitschrift Sezession, der - gut erkennbar im Briefwechsel mit dem Soziologen Armin Nassehi - sein politisches Programm als ,bewahrend' darstellt und formuliert: „Die Gruppenexistenz des ,Wir‘ im nationalen und damit auch ethnisch gebundenen Sinn ist unhintergehbar, davon bin ich überzeugt." (Brief v. 8.4.2014, in: Nassehi 2015: 320, vgl. Grimm 2016) Was also konserviert werden soll, ist die ahistorische Vorstellung eines ,traditionell geschlossenen, organisch strukturierten und homogenen gesellschaftlichen Raums, der keine Leere zulässt" (Žižek 2001: 259). Weil diese essentialistische heile deutsche Welt jedoch eine historische Fiktion ist, verschleiert das Etikett konservativ den aggressiven und immer schon (kultur-)rassistischen Ausschluss und die Umdeutung des Fremden zum Feind (vgl. Schmitt 1987 [1932]).

Eine andere problematische Diskussion zu treffenden Bezeichnungen für rechte Bewegungen und Akteure verläuft entlang der Differenz zwischen 
alter und Neuer Rechter. Während die alte Rechte, so die etablierte Unterscheidung, auf klassischen Rassentheorien basiert und den Nationalsozialismus glorifiziert, sei die Neue Rechte davon abgerückt, wie sogenannte Rechtsintellektuelle gern behaupten (vgl. Langebach/Raabe 2016). Stattdessen zieht die Neue Rechte nun essenzialisierte kulturelle Grenzen und erweckt mit dem Begriff ,Ethnopluralismus' mehr oder weniger erfolgreich den Anschein, Vielfalt nicht nur zu tolerieren, sondern sogar zu feiern. Womit sie im Übrigen in Einklang steht mit der Entwicklung des rassistischen Wissens insgesamt. Diese Differenzierung von Rassismus und kultureller Unterscheidung wird seit längerem seitens der Rassismusforschung und der antirassistischen Bewegungen (Müller 1995) zurückgewiesen, weil Kultur dabei zum ausgrenzenden Kampfbegriff wird, der innen von außen und zugehörig von nicht zugehörig trennt. Kultur umschreibt dann keinen „bloß impliziten Hintergrund alltäglicher Praxis“ mehr (Reckwitz 2017: 400), sondern wird stattdessen naturalisiert und essenzialisiert. Kurz: Man sagt Kultur statt Rasse, meint aber dasselbe (vgl. Kalpaka/Räthzel 2000: 179ff., Kalpaka 2005). Es handelt sich in jedem Fall um die Vorstellung einer quasinatürlichen, authentischen und fest an einen geographischen Raum gebundenen Kultur, was den Unterschied zum traditionellen Rassismus praktisch einebnet.

Andererseits ist der Name,Neue Rechte' eine Eigenbezeichnung, abgeleitet von der französischen ,Nouvelle Droite und propagiert von rechten Denkern wie Alain de Benoist oder Armin Mohler. Die deskriptive Verwendung des Konzepts läuft also Gefahr, die Propaganda jener Gruppierungen zu reproduzieren, die sich allzu gerne als rebellische Herausforderer einer alten Ordnung, eines Establishments oder vermeintlich linker Eliten inszenieren. Ohne Zweifel unterscheiden sich die Neuen Rechten von ihren Vorgängern hinsichtlich ihrer Formen politischer Mobilisierung und mancher rhetorischer Wendungen. Die Adaption des Begriffs würde aber bedeuten, die Abgrenzung von einer untrennbar mit dem Nationalsozialismus verbundenen ,alten Rechten' zu reproduzieren, die einer kritischen Analyse nicht standhält (vgl. Weiß 2017).

Neben der Frage, wer die Rechten sind, ist eine andere nicht minder drängend: Wie ist ihr Aufstieg spätestens seit 2015 zu erklären? Verschiedene Deutungen sind im Umlauf. Erstens und der Vollständigkeit halber sei jener falsche Erklärungsansatz erwähnt, den die Rechten selbst anführen würden: Dass sie reale und legitime Bedürfnisse befriedigen würden, da Zuwanderung zu ökonomischen Problemen und einer schwierigen Sicherheitslage führe und der Zulauf zu rechten Parteien eine rationale Reaktion auf diese Entwicklungen sei. In Europa oder den USA nehmen diese Argumente im Kontext rechter Rhetorik eine zentrale Stellung ein und beeinflussen den politischen Mainstream (vgl. Metz/Seeßlen 2016). Die wirtschaftlichen oder kriminalitätsbezogenen Zahlen zeigen jedoch deutlich, wie weit die Realität von rechter Fiktion und Inszenierung entfernt ist. Ohnehin sind rassistische Einstellungen und rechte Wahlpräferenzen gerade an Orten verbreitet, die eine hohe ethnische Homogenität aufweisen und an denen Zuwanderung von Ausländer_innen kaum stattfindet (vgl. Benrath/Giesel 2018). Hinzu kommt, dass die erwähnte Annahme einer vernünftigen Reaktion auf eine angeblich tatsächliche Bedrohung immer schon mit dem bereits beschriebenen 
ahistorischen Bild homogener und räumlich geschlossener Gesellschaften spielt, die es zu verteidigen gelte. Wer sich auf diese Argumentationslinie einlässt, fällt also zugleich auf die von rechter Agitation befeuerte Täter-OpferUmkehr herein - auf die Behauptung also, die deutsche Bevölkerung etwa sei das Opfer einer ,Umvolkung' und rechte Gewalt sei nur Selbstverteidigung.

Die empirischen Differenzen zwischen rechter Fiktion und Wirklichkeit deuten bereits an, dass ein simples Reiz-Reaktions-Schema als Erklärung für politische Präferenzen zu kurz greift. Politikwissenschaftliche Studien, die wir einem zweiten Erklärungsansatz zuordnen, untersuchen demgegenüber statistisch signifikante Korrelationen zwischen Wahlentscheidungen und sozioökonomischen Merkmalen. Dabei erklären einige, dass die AfD unter jenen mit geringen Einkommen und niedrigem sozialem Status auf überdurchschnittliche Zustimmung stößt (vgl. Vehrkamp/Wegschaider 2017, Lux 2018, Tutić/von Hermanni 2018). Daraus wird die eingangs erwähnte Variation der Abgehängten- oder Notwehr-These abgeleitet: Sozioökonomisch Benachteiligte würden aus Protest gegen eine (neo-)liberale Ordnung rechte Parteien wählen. Von diesen erhofften sie sich zwar nicht unbedingt Umverteilung, aber zumindest die Sicherung ihres Besitzstandes und ihrer Privilegien gegenüber Migrant_innen, dievor allem als Konkurrent_innen wahrgenommenen werden. Aus dieser Annahme einer „Rückkehr des Hauptwiderspruchs“ (kritisch dazu Dowling et al. 2017) wird die als realistisch und pragmatisch bezeichnete Forderung abgeleitet, dass das Soziale wieder im Zentrum stehen müsse. Diese sozialpolitische Logik grenzt sich von kulturell konnotierten und als identitätspolitisch umschriebenen Kämpfen etwa gegen Sexismus und Rassismus ab, die - verkürzt wiedergegeben - eine tendenziell als weiß und männlich konzipierte Stammwählerschaft in die Arme der Rechten getrieben hätten (vgl. zu den USA insbesondere Lilla 2017, zu Deutschland v. a. Nölke 2017, Heisterhagen 2018). Diese Differenzen zwischen der Betonung sozioökonomischer Faktoren einerseits und einem intersektional und global gedachten Gerechtigkeitsideal andererseits provoziert eine scharf geführte wissenschaftliche und politische Debatte.

Mit der letztgenannten Position korrespondiert in gewisser Weise der dritte Komplex an Erklärungsansätzen für die Erfolge rechter Parteien. Dieser betont gegenüber der Feststellung, dass die AfD unter Menschen mit geringeren Einkommen auf größere Zustimmung stoße, dass deren Anhängerschaft sich insgesamt dennoch zu auffällig großen Anteilen aus Angehörigen mittlerer und hoher Einkommens- und Statusgruppen zusammensetze (vgl. Lengfeld 2017). Die Mathematik von Teilmengen bringt es zwar mit sich, dass beide Aussagen rechnerisch zutreffend sein können, jedoch zeigt eine weitere Studie auf Grundlage der Daten des Sozio-Oekonomischen Panels (SOEP), dass „AfD-Unterstützer sich unabhängig von ihrer wirtschaftlichen Situation und sonstigen Einstellungen vor allem durch eines auszeichnen: Sie wollen nicht, dass Flüchtlinge nach Deutschland einwandern, weil sie deren Einfluss kritisch sehen." (Schröder 2018: 2, vgl. auch Lengfeld/Dilger 2018) Zugespitzt gesagt: Eine Partei, die eine rassistische Politik vertritt, werde von Menschen mit rassistischen Einstellungen gewählt. Alle anderen Faktoren seien zweitrangig bis unbedeutend.

Wir halten die Gegenüberstellung von Identitätspolitik (einer an Klassenwidersprüchen angeblich uninteressierten Linken) und 
Klassenpolitik (einer gegenüber Rassismus und Homophobie ignoranten Arbeiterklasse) für nicht hilfreich. Vermeintliche Minderheitenprobleme wie rassistische Diskriminierung oder Sexismus betreffen natürlich auch Arbeiter_innen, und Deklassierte haben auch Identitäten. Von der „notwendigen Zusammenführung von Klassenpolitik und emanzipatorischer Identitätspolitik“ (van Dyk 2019: 31) wird zwar häufig gesprochen, im Grunde ist die Debatte aber noch im Stadium der Trennung und Schuldzuschreibung.

Eine weitere Spur, wie der Aufstieg der Rechten zu erklären sein könnte, findet sich in raumbezogenen Analysen, die dieser Themenschwerpunkt aufgreift. Wie eingangs angedeutet, stechen messbare Differenzen zwischen Ost und West beziehungsweise zwischen Stadt und Land hervor und dominieren die Debatte. Daher ist die Frage nicht abwegig, ob es überhaupt eine ,Stadt von rechts' gibt. Schließlich gilt die Stadt gemeinhin als Ort sozialer und demographischer Dynamik, als Ort der Zuwanderung und der Begegnungen zwischen Fremden (vgl. Simmel 1903). Ist die Erfahrung von Unterschiedlichkeit für die Großstädter_innen nicht eine Schule der Toleranz? Einwände dieser Art sind zu erwarten, wenn in einer Zeitschrift für kritische Stadtforschung über den Rechtsruck diskutiert werden soll. Im Grunde genommen kreisen sie alle um die Vorstellung, dass das Leben in der Großstadt mit rechten Haltungen mehr oder weniger unvereinbar sei oder zumindest eine größere Widerstandsfähigkeit dagegen aufweise. In diesem Sinne formuliert etwa der Architekt Friedrich von Borries (2016: 181): „Die offene Gesellschaft braucht die Stadt, und die Stadt der Gegenwart braucht die offene Gesellschaft."

Diese Annahme stützt sich auf Wahlergebnisse - vorausgesetzt, dass rechte Orientierungen sich an der Wahl einer rechten Partei ablesen lassen. Bei der Bundestagswahl 2017 hat die AfD in den Großstädten der gesamten BRD jedenfalls weniger Stimmen erhalten als im ländlichen Raum. Von Leipzig und Dresden abgesehen (18,3 und 22,5 Prozent), blieb die Partei in allen Großstädten mit über 250.00o Einwohner*innen unter dem Bundesdurchschnitt (12,6 Prozent). Die höchsten Ergebnisse konnte sie hingegen abseits der Stadtregionen erzielen, etwa im Osterzgebirge (35,5 Prozent) oder im bayrischen Deggendorf (19,2 Prozent). Bei genauerer Betrachtung bieten die Wahlergebnisse allerdings kein so klares Bild. Denn innerhalb großstädtischer Räume lassen sich teils erhebliche Differenzen erkennen. In Leipzig etwa reicht die Spannweite von 7,7 Prozent in der zentrumsnahen Südvorstadt bis 29,5 Prozent in der peripher gelegenen Großwohnsiedlung Grünau oder 27,8 Prozent in der suburbanen Eigenheimsiedlung AlthenKleinpösna. Ähnliche Differenzen lassen sich auch in Dortmund und anderen Städten in den westlichen Bundesländern finden.[3] Wie auch immer sich diese Ergebnisse weiter deuten lassen: Wahlgeographisch lässt sich der Rechtspopulismus nicht einfach den Dörfern und Kleinstädten zuschieben. Einschlägige Studien kommen zum Schluss, dass der Unterschied in der Verteilung rechtspopulistischer, rechtsextremer und neurechter Einstellungen zwischen Stadt und Land „nicht sehr groß“ (Küpper 2017: 31) sei. Einige Beiträge des Hefts (von Förtner et al., Bernet und Intelmann) greifen die Frage nach der Aussagekraft (wahl-)statistischer Daten auf und vertiefen die raumbezogenen Überlegungen.

Ein weiterer Einwand gegen die Frage nach ,Stadt von rechts' stützt sich auf die traditionelle und gut erforschte Großstadtfeindschaft der Rechten 
(vgl. Höhne 2015): Wie sollen sich rechte Orientierungen, deren Merkmal doch gerade die Ablehnung all dessen ist, was man mit großstädtischen Lebensformen assoziiert, in den Metropolen nachhaltig verfestigen können? Auch dieses Argument lohnt einen zweiten Blick. Schon das im ausgehenden 18. Jahrhundert in Europa entstehende moderne Städtewesen zog Ressentiments von Agrariern, Landadel und Besitzbürgertum auf sich. Die Großstadtfeindschaft, die politische (Proletariat), moralische (Sittenverfall) und psychologisierende (Reizüberflutung) Stereotype bemühte, wurde durch die Nationalsozialisten völkisch-rassistisch zugespitzt. Der Nationalsozialismus zielte auf die Ansiedlung einzelner Familien im ländlichen Raum, auf Kleinstbesitz und Selbstversorgung. Die programmatisch verkündete Zerschlagung der Großstädte (vgl. Schubert 1986: 35) war jedoch schon aufgrund der Anforderungen der Rüstungsindustrie nicht umsetzbar. Zudem zeigte sich der nationalsozialistische Größenwahn auch in seinen Bauvorhaben und städtischen Arrangements, etwa der Planung der neuen gigantischen Reichs- oder Welthauptstadt Germania. Aber wenn schon nicht die Großstadt, so sollte zumindest Urbanität als Lebensform auf der Grenze von „Ordnung und Chaos“ (Siebel 2012) zugunsten geordneter und kontrollierter Städte abgeschafft werden. Insofern hat die sogenannte ,alte Rechte durchaus eine Form von Metropolenpolitik betrieben, nicht zuletzt bezüglich der in den 1930er Jahren drängenden Wohnungsfrage. Untersuchungen zu dieser Politik zeigen etwa, wie der Verteilungskampf um knappen Wohnraum auf dem Rücken von sogenannten „Gemeinschaftsfremden“ und „Reichsfremden“ ausgetragen wurde (Haerendel 1991: 869). Es schließt sich die Frage an, wie rechte Parteien und Bewegungen heute auf die Großstädte und ihre Probleme reagieren.

Damit ist einem weiteren Einwand der Weg bereitet, der gegen unsere Frage nach einer möglichen ,Stadt von rechts‘ vorgebracht werden könnte: Oft ist zu hören, es sei nichts Neues, dass Rechte auch in Städten unterwegs sind. Ohne Zweifel sind rechte Haltungen auch in urbanen Räumen zu finden und die Rechten wollen die Metropolen nicht per se auflösen, sondern haben eigene Vorstellungen einer rechten (segregierten und durchorganisierten) Stadt. Aber gibt es spezifische Gründe, den Rechtsruck aus der Perspektive einer kritischen Stadtforschung ins Visier zu nehmen, die sich mit neoliberalen Transformationen, sozialer Polarisierung, Aufwertung und Verdrängung beschäftigt? Auf die Wohnungsfrage bezogen, könnte man entwarnend meinen: In den zentrumsnahen Lagen der Großstädte, den Hotspots der Gentrifizierung, spielen Rechte eine eher moderate Rolle. Trotz zunehmender Einmischung der AfD in stadtpolitische Themen wie Gentrifizierung sind jene Quartiere deutscher Großstädte, in denen sich Aufwertung und Mietsteigerung konzentrieren, nicht jene Quartiere, in denen die AfD ihre Stimmengewinne einfahren konnte; häufig ist das Gegenteil der Fall.[4] Der Rechtsruck scheint, so gesehen, ein gutes Stück von den Problemen der Stadtentwicklung, sofern sie sich in der Wohnraumkrise verdichten, entfernt. Auch hier lässt sich dagegenhalten: Erstens sind Wahlergebnisse nur ein Indikator; rechte (oder progressive) Haltungen müssen auch jenseits von Wahlergebnissen untersucht werden. Zweitens sind - exemplarisch gesprochen - die 14 Prozent der Zweitstimmen, die die AfD im wohnungsmarktbezogen dynamischen Leipziger Osten geholt hat, Grund genug, nach 
dem Zusammenhang von Aufwertung und Rechtsruck zu fragen. Drittens wäre auch zu untersuchen, ob nicht gerade unter denen, die bereits in die peripheren Quartiere verdrängt wurden, problematische (personalisierende, rassistische) Deutungen ihrer prekären Wohnsituation verbreitet sind. Viertens wäre zu prüfen, ob Ressourcenkonflikte etwa um Wohnraum oder Kita-Plätze und Abstufungen zwischen vermeintlich besseren und schlechteren Quartieren (Ausstattung von Schulen, Hierarchien in der Sanierungsfolge öffentlicher Einrichtungen etc.) rechte Deutungsmuster aktivieren, die im Alltagsverstand vorhanden sind.

Der Rechtsruck steht, entgegen aller Beschwichtigungsversuche, in engem Zusammenhang mit sozialräumlichen Entwicklungen in Städten. Wie Zentrum und Peripherie innerhalb und außerhalb der Städte die Zunahme und Enttabuisierung rechten Denkens beeinflussen, ist eine spannende Frage, der sich der Schwerpunkt widmet.

\section{Die Beiträge des Schwerpunkts}

Maximilian Förtner, Bernd Belina und Matthias Naumann werfen in ihrem Beitrag „Stadt, Land, AfD“ ganz grundsätzlich das bereits angesprochene Problem auf, dass die meist starr verstandene und in räumlichen Arrangements gegossene Differenzierung von Stadt und Land zu kurz greift. Stattdessen unterscheiden sie zwischen Urbanität und Ruralität, die jeweils als Praxis der Raumproduktion verstanden und sozioökonomisch rückgekoppelt werden. Dadurch entsteht ein komplexeres Bild von geographischen und sozialen Räumen, die unterschiedlich hervorgebracht werden und deren wesentliche Differenz jene zwischen Zentrum und Peripherie ist, die bisweilen quer zu der von Stadt und Land liegen kann. Diese Perspektive hat den Vorteil, dass periphere Zentren - also rurale Lebenswelten im städtischen Umfeld - und zentrale Peripherien - also mehr oder weniger abgehängte Städte in eher ruralen Regionen - lesbar werden. Der Beitrag stellt drei in diesem Kontext idealtypische Räume vor. Dies provoziert neue, spannende Erkenntnisse im Hinblick auf die Wahlgeographien der Rechten.

Tobias Bernets Text „Stadtstaaten oder Barbarei?“ lässt sich ähnlich lesen. Bernet hebt hervor, dass - in den USA wie in Deutschland - die Binnenmigration ein viel größeres Thema ist beziehungsweise sein müsste als jene von außen. Die Abwanderung aus ländlichen oder peripheren Regionen vor allem in sogenannte Schwarmstädte - also in jene Zentren, die am ehesten das Etikett urban verdienen - ist deutlich messbar und verschärft sozioökonomische und kulturelle Konflikte zwischen Zentren und Peripherien. Daher bedarf es mehr denn je einer inklusiven und egalitären Bewegung für ein ,Recht auf Stadt', nicht nur gegen die autoritär-exkludierenden Kräfte von rechts, sondern auch gegen eine mit diesen oft genug verschränkte neoliberale bis neofeudale Immobilienwirtschaft.

Einen anderen Weg schlägt Nikolai Huke ein. Sein Beitrag „Die neue Angst vorm schwarzen Mann“ diskutiert Moralpaniken im Kontext verschiedener räumlicher Bezüge, also die affektive und kollektive Konstruktion des Fremden als Feind. Ein permanent reproduzierter Generalverdacht in Kombination mit assoziativen Äquivalenzketten und demagogisch-rassistischen Sprachmustern stellen den Fremden erst her. Die Art und Weise der 
Kommunikation in sozialen Medien verstärkt diese Dynamik. Ihren Niederschlag findet dieses Othering, wie der Autor zeigt, oftmals in der Verknüpfung der konstruierten Gruppen mit bestimmten Räumen (Problemviertel, Angsträume etc.) und Raumpolitiken.

Während der Begriff,Moralpanik übersteigerte oder gar inszenierte Ängste umschreibt, nehmen Jan Üblacker und Tim Lukas eine andere Art von Angst oder Sorge in den Blick. Sie untersuchen in ihrem Beitrag „Keine Angst, es ist nur Gentrification?" die teils drastischen Veränderungen im Düsseldorfer Bahnhofsviertel. Die immobilienwirtschaftliche und politische Aufwertung von Quartieren führt zu Verdrängungsdruck und sozialen Abstiegsängsten der Bewohner_innen. Hinzu kommt, dass besonders Bahnhofsquartiere von vermeintlichen und tatsächlichen Risiken und Unsicherheitserwartungen betroffen sind, die sich zur politischen Instrumentalisierung eignen. Daraus ist zwar noch kein Mechanismus beziehungsweise kein Automatismus nach rechts abzuleiten. Dennoch legen die Befunde einen möglichen Zusammenhang oder mindestens einen Problemhorizont nah.

In der Debatte gehen wir die Thematik mit einer grundlegenden forschungspraktischen Frage an: Sollte man - in der Forschung - mit Rechten reden, und wenn ja, wie? Sollen wir in unseren Erforschungen rechter politischer Entwicklungen mit Rechten beziehungsweise politisch rechts handelnden Menschen Kontakt aufnehmen? Wiekönnen die Interaktionen aussehen, welche Zugänge können wir wählen? Wie positionieren wir uns selbst innerhalb dieser Forschung? Den Debattenaufschlag von Robert Feustel diskutieren Regina Ammicht Quinn, Lee Hielscher, Annekatrin Kühn und Katrin Lehn, Nils Kumkar, Daniel Mullis und Andreas Nölke. Ebenfalls als Beitrag in der Rubrik Debatte erscheint der Text „Sieben Thesen zur urbanen Krise in Chemnitz"von Dominik Intelmann. Ausgehend von den Ereignissen in Chemnitz im Sommer 2018 plädiert der Autor für eine ortssensible Perspektive, bei der die Analyse der lokalen Stadtgesellschaft die historischen und gesellschaftlichen Spezifika ostdeutscher Erfahrungen berücksichtigt.

Im Magazinteil setzt sich Christian Marty mit der Rezeption Ernst Jüngers durch die Identitäre Bewegung auseinander, einer Bewegung, die sich dezidiert als eine urbane und akademische Jugendbewegung von rechts inszeniert. Damit rückt Marty vor dem Hintergrund einer profunden Kenntnis Ernst Jüngers die Frage in den Blick, wie rechtes Denken über die Stadt versucht, sich jenseits großstadtfeindlicher Ressentiments eine Geschichte und Vordenker zu geben und zeigt, wie dieses Vorhaben scheitert.

Ebenfalls im Magazin widmet sich Magdalena Augustin einem weiteren Feld rechter Stadtpolitik, das bislang noch wenig Beachtung gefunden hat. In ihrem Beitrag „Vom Denkmalschutz zum Heimatschutz“ untersucht sie anhand von zwei Wiener Beispielen und von Veröffentlichungen der FPÖ rechte Deutungsmuster im lokalen Diskurs um Denkmalschutz.

Während in den bisher genannten Beiträgen rechte Akteure im Zentrum des Interesses standen, plädiert Heike Kleffner in ihrem Beitrag „Wider die Unsichtbarkeit“ eindringlich dafür, mit Betroffenen rechter Gewalt zu sprechen. Sie liefert Argumente für die Notwendigkeit, Betroffene rechter Gewalt im städtischen Raum sichtbar zu machen. Die Gruppe critique'n'act stellt in ihrem Beitrag „Neoliberale Stadt und rechte Hegemonie?“ am Beispiel der 
Stadt Plauen Wege vor, wie emanzipatorische Kräfte der Region gestärkt werden können. Das Interview mit einem Aktivisten einer Berliner AntifaGruppe thematisiert die Gegebenheiten vor Ort (Berliner Nordosten) und ihre Bedeutung sowohl für autoritäre und rechte Bewegungen als auch für antifaschistischen Protest. Geschichte, Sozialstruktur und Veränderungen des Straßenbilds, der Nachbarschaften et cetera im Großbezirk Pankow werden als Ausdruck und als Bedingung rechter Hegemoniebestrebungen erkennbar. Der Interviewpartner gibt Auskunft über Strategien und Forderungen, mit denen sich rechte Akteure in lokale Auseinandersetzungen um Sicherheit, Wohnungen oder Großprojekte einbringen und verdeutlicht zudem das Verhältnis von antifaschistischer und wohnungspolitischer, also sozialer Bewegung.

Zwei Rezensionen folgen. Ina Schildbach bespricht Arbeiterbewegung von rechts, herausgegeben von Karina Becker, Klaus Dörre und Peter ReifSpirek. Und Jan Lange diskutiert das Buch Städte im Nationalsozialismus, herausgegeben von Winfried Süß und Malte Thießen.

Einige Lücken und Leerstellen fallen auf und sollen nicht unerwähnt bleiben. Es gibt Themen und Stimmen, die zu kurz oder gar nicht zur Sprache kommen. An erster Stelle fällt die Abwesenheit von People of Color und damit von Menschen auf, die von den hier thematisierten Praktiken und Raumnahmen rechter Akteure in besonderem Maße betroffen sind. Rassismuserfahrungen werden zwar im Beitrag von Heike Kleffner ins Zentrum gerückt, wir hätten uns aber gewünscht, dass es uns gelingt, einer Auseinandersetzung damit mehr Raum zu geben.

Leider ist es nicht das erste Mal, dass die Rubrik der Aufsätze in diesem Heft ausschließlich von Männern bestritten wird. Dies hat, denken wir, Auswirkungen auf die Inhalte und verdeutlicht die Strukturen des Wissenschaftssystems (sowie geschlechtsspezifisch geprägte Themenfelder). Das ist problematisch, nicht zuletzt für eine kritische Zeitschrift. Schließlich: Wenn diese Einleitung damit beginnt, dass wir von einem weltweiten Rechtsruck ausgehen, so wäre es wichtig, die Fragen einer Stadt von rechts auch anhand von Beispielen jenseits des deutschsprachigen Raumes zu diskutieren. Andere geographische Kontexte wären hochinteressant und kommen zu kurz. Gerade angesichts dieser Leerstellen wünschen wir uns daher, dass dieser Themenschwerpunkt weitere Diskussionen anstößt, die wir auch hier in sub \urban weiter führen wollen.

Die Publikation dieses Beitrags wurde durch Mittel des Open-AccessPublikationsfonds der Freien Universität Berlin gefördert.

\section{Endnoten}

[1] Viele wichtige Beiträge finden sich in den letzten Ausgaben der Zeitschrift Prokla, u. a. Lessenich (2017), Opratko (2017), Eversberg (2018), Sablowski/Thien (2018); mit Stadt-/Raumbezug Belina (2017), Bescherer (2019). Vgl. mit Bezug auf den digitalen Kapitalismus außerdem Reitz (2017).

[2] Das Forschungsvorhaben an den Universitäten Jena und Tübingen wird gefördert vom BMBF. Genauere Angaben unter www.podesta-projekt.de. 
[3] Die Stimmenverteilung bundesweit ist unter www.bundeswahlleiter.de dokumentiert, die Ergebnisse in den erwähnten Städten und Regionen sind zu finden unter: www.leipzig.de, www.statistik.sachsen.de, www.landkreis-deggendorf.de) (letzter Zugriff am 11.4.2019).

[4] Im Berliner Wahlbezirk Friedrichshain-Kreuzberg-Prenzlauer Berg erhielt die AfD bei der Bundestagswahl 2017 6,3 Prozent der Zweitstimmen, der niedrigste Wert im Stadtvergleich; Neukölln kommt mit 11,2 Prozent auf die Hälfte des Spitzenwerts (21,6 Prozent in Marzahn-Helldesdorf), vgl. https://www.wahlen-berlin.de/wahlen/BU2017/afspraes/ zweitstimmen_wahlkreis-75-berlin-mitte_gesamt.html (letzter Zugriff am 11.4.2019). In Hamburg-Sternschanze erhielt die Partei 2,3 Prozent der Zweitstimmen, in St. Pauli 4,5 Prozent bei stadtweit 7,8 Prozent, vgl. https://www.statistik-nord.de/fileadmin/ Dokumente/Wahlen/Hamburg/Bundestagswahlen/2017/Wahlergebnisse/endgültig/ most_important/BTW_2017_HH_END_INTERNET.pdf(letzter Zugriff am 11.4.2019). Ähnlich verhält es sich in München und Köln.

\section{Autor_innen}

Tobias Bernet ist Historiker und Ethnologe und forscht zu Stadtentwicklung, Wohnungspolitik und sozialen Bewegungen.

mail@tobiasbernet.de

Peter Bescherer ist Soziologe. Seine Forschungs- und Interessenschwerpunkte sind Stadtforschung, Demokratietheorie, soziale Bewegungen und Arbeitssoziologie.

peter.bescherer@uni-jena.de

Kristine Beurskens ist Geographin und erforscht politische und emotionale Geographien von Sicherheit und Grenzen.

k_beurskens@ifl-leipzig.de

Robert Feustel ist Politikwissenschaftler und beschäftigt sich mit politischer Theorie sowie Wissens- und Stadtsoziologie.

robert.feustel@uni-jena.de

Boris Michel ist Geograph. Er ist Teil der Redaktion von sub \urban, arbeitet zu Geschichte der Geographie und beschäftigt sich mit Stadtgeographie zurzeit hauptsächlich in der Lehre. boris.michel@fau.de

\section{Literatur}

Adorno, Theodor W. (1977 [1967]): Erziehung nach Auschwitz. In: Gesammelte Schriften Bd. 10.2. Frankfurt am Main: Suhrkamp, 674-690.

Becker, Anna / Göppert, Hannah / Schnur, Olaf / Schreiber, Franziska (2018): Die digitale Renaissance der Nachbarschaft. Soziale Medien als Instrument postmoderner Nachbarschaftsbildung. In: Forum Wohnen und Stadtentwicklung 4/2018, 206-210.

Belina, Bernd (2017): Zur Geographie der Abstiegsgesellschaft. In: Prokla. Zeitschrift für kritische Sozialwissenschaft 47/1, 97-104.

Benrath, Bastian / Giesel, Jens (2018): Kennen AfD-Wähler Ausländer? https://www.faz. net/aktuell/wirtschaft/wirtschaft-in-zahlen/deutschlandkarte-auslaenderanteil-vs-afdwaehler-infografik-15740830.html (letzter Zugriff am 11. 4. 2019).

Bescherer, Peter (2019): „Stadt von rechts“ oder „Recht auf Stadt“? In: Prokla. Zeitschrift für kritische Sozialwissenschaft 49/1, 157-161.

Bonikowski, Bart / Halikiopoulou, Daphne / Kaufmann, Eric / Rooduijn, Matthijs (2019): Populism and nationalism in a comparative perspective: a scholarly exchange. In: Nations and Nationalism 25/1, 58-81.

Borries, Friedrich von (2016): Die Stadt der offenen Gesellschaft. In: Harald Welzer (Hg.), Die offene Gesellschaft und ihre Freunde. Frankfurt am Main: Fischer, 180-185. 
D’Antonio, Oliver (2017): Stadt, Land, Partei - neue Asymmetrien im Parteienwettbewerb? In: Sebastian Bukow / Uwe Jun (Hg.), Parteien unter Wettbewerbsdruck. Wiesbaden: Springer VS, 123-150.

Dörre, Klaus / Bose, Sophie / Lütten, John / Köster, Jakob (2018): Arbeiterbewegung von rechts? Motive und Grenzen einer imaginären Revolte. In: Berliner Journal für Soziologie 28/1-2, 55-89.

Dowling, Emma / van Dyk, Silke / Graefe, Stefanie (2017): Rückkehr des Hauptwiderspruchs? Anmerkungen zur aktuellen Debatte um den Erfolg der Neuen Rechten und das Versagen der „Identitätspolitik“. In: Prokla. Zeitschrift für kritische Sozialwissenschaft 47/3, 411-420.

Eckardt, Frank (2018): Willkommene Flüchtlinge, ungeliebte Nachbarn. In: Nina Berding / Wolf-Dietrich Bukow / Karin Cudak (Hg.), Die kompakte Stadt der Zukunft. Wiesbaden: Springer VS, 141-157.

Eversberg, Dennis (2018): Innerimperiale Kämpfe. In: Prokla. Zeitschrift für kritische Sozialwissenschaft 48/1, 43-54.

Feustel, Robert / Bescherer, Peter (2018): Der doppelte Populismus. Konturen eines schwierigen Begriffs. In: Berliner Debatte Initial 29/2, 133-144.

Forum für kritische Rechtsextremismusforschung (FKR) (Hg.) (2011): Ordnung. Macht. Extremismus. Effekte und Alternativen des Extremismus-Modells. Wiesbaden: Springer VS.

Grimm, Rico (2016): Neue Rechte. „Wiewohl ich skeptisch bin, will ich wenigstens in dieser Weise offen sein“. krautreporter.de/1284--wiewohl-ich-skeptisch-bin-will-ichwenigstens-in-dieser-weise-offen-sein (letzter Zugriff am 20. 2. 2019).

Haerendel, Ulrike (1999): Wohnungspolitik im Nationalsozialismus. In: Zeitschrift für Sozialreform 45/10, 843-879.

Heisterhagen, Nils (2018): Die liberale Illusion: Warum wir einen linken Realismus brauchen. Bonn: Dietz.

Heitmeyer, Wilhelm (2018): „Der Begriff ,Rechtspopulismus“ ist viel zu verharmlosend“. https://www.deutschlandfunkkultur.de/soziologe-wilhelm-heitmeyer-der-begriffrechtspopulismus.990.de.html?dram:article_id=432919 (letzter Zugriff am 12.3.2019).

Hillje, Johannes (2018): Rückkehr zu den politisch Verlassenen. Gespräche in rechtspopulistischen Hochburgen in Deutschland und Frankreich. http://www.progressiveszentrum.org/die-verlassenen/ (letzter Zugriff am 11. 4. 2019).

Höhne, Stefan (2015): Die Idiotie des Stadtlebens. In: Zeitschrift für Ideengeschichte 9/2, 39-46.

Kalpaka, Annita (2005): Pädagogische Professionalität in der Kulturalisierungsfalle - Über den Umgang mit ,Kultur' in Verhältnissen von Differenz und Dominanz. In: Rudolf Leiprecht / Anne Kerber (Hg.), Schule in der Einwanderungsgesellschaft. Ein Handbuch, Schwalbach: Wochenschau, 387-405.

Kalpaka, Annita / Räthzel, Nora (2000): Die Schwierigkeit, nicht rassistisch zu sein. In: Nora Räthzel (Hg.), Theorien über Rassismus. Hamburg: Argument, 177-190.

Kaschuba, Wolfgang (2016): Die Rache der Dörfer. https://www.deutschlandfunkkultur. de/rechtspopulismus-die-rache-der-doerfer.10o8.de.html?dram:article_id=371289 (letzter Zugriff am 11. 4. 2019).

Keller, Luisa / Berger, David (2017): Pegida entdemokratisiert - zur Instrumentalisierung von Angst im öffentlichen Raum. In: Tino Heim (Hg.), Pegida als Spiegel und Projektionsfläche. Wiesbaden: Springer VS, 307-340.

Küpper, Beate (2017): Rechtspopulismus im ländlichen Raum. In: Ländlicher Raum 68/2, 30-31.

Laclau, Ernesto (2005): On Populist Reason. London/New York: Verso.

Langebach, Martin / Raabe, Jan (2016): Die „Neue Rechte in der Bundesrepublik Deutschland. In: Fabian Virchow / Martin Langebach / Alexander Häusler (Hg.), Handbuch Rechtsextremismus. Wiesbaden: Springer VS, 561-592.

Lengfeld, Holger (2017): Die „Alternative für Deutschland“: eine Partei für Modernisierungsverlierer? In: Kölner Zeitschrift für Soziologie und Sozialpsychologie 69/2, 209-232.

Lengfeld, Holger / Dilger, Clara (2018): Kulturelle und ökonomische Bedrohung: Eine Analyse der Ursachen der Parteiidentifikation mit der „Alternative für Deutschland“ mit dem Sozio-oekonomischen Panel 2016. In: Zeitschrift für Soziologie 47/3, 181-199.

Lessenich, Stephan (2017): Probleme der Klassenanalyse. In: Prokla. Zeitschrift für kritische Sozialwissenschaft, 47/1, 111-115. 
Lilla, Mark (2017): The Once and Future Liberal: After Identity Politics. New York: HarperCollins.

Lux, Thomas (2018): Die AfD und die unteren Statuslagen: Eine Forschungsnotiz zu Holger Lengfelds Studie Die „Alternative für Deutschland“: eine Partei für Modernisierungsverlierer? In: Kölner Zeitschrift für Soziologie und Sozialpsychologie 70/2, 255-273.

Marchart, Oliver (2017): Liberaler Antipopulismus. Ein Ausdruck von Postpolitik. In: Aus Politik und Zeitgeschichte 67/44-45, 11-16.

Metz, Markus / Seeßlen, Georg (2016): Hass und Hoffnung. Deutschland. Europa und die Flüchtlinge. Berlin: Bertz+Fischer.

Müller, Jost (1995): Mythen der Rechten. Nation, Ethnie, Kultur. Berlin/Amsterdam: ID-Archiv.

Nassehi, Armin (2015): Die letzte Stunde der Wahrheit. Hamburg: Murmann.

Nölke, Andreas (2017): Linkspopulär: Vorwärts handeln statt rückwärts denken. Frankfurt am Main: Westend.

Olschanski, Reinhard (2017): Der Wille zum Feind. Paderborn: Fink.

Opratko, Benjamin (2017): Rechtspopulismus als Krisenbearbeitung. In: Prokla. Zeitschrift für Kritische Sozialwissenschaft 47/1, 123-130.

Oßenbrügge, Jürgen (2018): „March for Sozialgeographie“? Rechtspopulismus als Zumutung und die regressive Moderne als Herausforderung der Humangeographie. In: Geographica Helvetica 73/4, 309-319.

Rancière, Jacques (2010): Der Hass der Demokratie. Berlin: August.

Rancière, Jacques (2017): Der unauffindbare Populismus. In: Alain Badiou / Pierre Bourdieu / Judith Butler / Georges Didi-Huberman / Sadri Khiari / Jacques Rancière, Was ist ein Volk? Hamburg: Laika, 97-101.

Reckwitz, Andreas (2017): Die Gesellschaft der Singularitäten. Zum Strukturwandel der Moderne. Berlin: Suhrkamp.

Reitz, Tilman (2017): Aufstand der Anteillosen? Autoritärer Protest im digitalen Kapitalismus. In: Das Argument 59/3, 363-377.

Riehl, Wilhelm Heinrich (1853): Die Naturgeschichte des Volkes als Grundlage einer deutschen Social Politik. Bd. 1: Land und Leute. Stuttgart: Cotta.

Sablowski, Thomas / Thien, Günther (2018): Die AfD, die ArbeiterInnenklasse und die Linke - kein Problem? In: Prokla. Zeitschrift für kritische Sozialwissenschaft 48/1, 55-72.

Schmitt, Carl (1987 [1932]): Der Begriff des Politischen. Berlin: Duncker \& Humblot.

Schröder, Martin (2018): AfD-Unterstützer sind nicht abgehängt, sondern ausländerfeindlich. SOEP-Papers 975. https://www.diw.de/documents/publikationen/73/ diw_01.c.595120.de/diw_spo975.pdf (letzter Zugriff am 12. 4. 2019).

Schubert, Dirk (1986): Großstadtfeindschaft und Stadtplanung. Neue Anmerkungen zu einer alten Diskussion. In: Die alte Stadt 13/1, 22-41.

Siebel, Walter (2012): Ordnung und Chaos. Bedingungen einer urbanen Stadt. In: Polar 12, 57-61.

Simmel, Georg (1995 [1903]): Die Großstädte und das Geistesleben. In: Gesamtausgabe Bd. 7. Frankfurt am Main, 116-131.

Spengler, Oswald (1918): Der Untergang des Abendlandes. Umrisse einer Morphologie der Weltgeschichte. Bd. 1: Gestalt und Wirklichkeit. Wien/Leipzig: Braumüller.

Spengler, Oswald (1922): Der Untergang des Abendlandes. Umrisse einer Morphologie der Weltgeschichte. Bd. 2: Welthistorische Perspektiven. München: Beck.

Spier, Tim (2016): Die Wahl von Rechtsaußenparteien in Deutschland. In: Fabian Virchow / Martin Langebach / Alexander Häusler (Hg.), Handbuch Rechtsextremismus. Wiesbaden: Springer VS, 257-284.

Stärck, Alexander (2018): Zur Rekonstruktion des Extremismusmodells. In: Philip Baron / Ansgar Drücker / Sebastian Seng (Hg.), Das Extremismusmodell. Düsseldorf: IDA, 20-26.

Tutić, Andreas / von Hermanni, Hagen (2018): Sozioökonomischer Status, Deprivation und die Affinität zur AfD - Eine Forschungsnotiz. In: Kölner Zeitschrift für Soziologie und Sozialpsychologie 70/2, 275-294.

van Dyk, Silke (2019): Identitätspolitik gegen ihre Kritik gelesen. Für einen rebellischen Universalismus. In: Aus Politik und Zeitgeschichte 68/9-11, 25-32. 
Vehrkamp, Robert / Wegschaider, Klaudia (2017): Populäre Wahlen: Mobilisierung und Gegenmobilisierung der sozialen Milieus bei der Bundestagswahl 2017. Gütersloh: Bertelsmann-Stiftung.

Weber, Max (1976 [1922]): Wirtschaft und Gesellschaft. Grundriß der verstehenden Soziologie. Tübingen: Mohr Siebeck.

Weiß, Volker (2017): Faschisten von heute? „Neue Rechte“ und ideologische Traditionen. In: Aus Politik und Zeitgeschichte 67/42-43, 4-9.

Wirz, Dominique S. (2018): Persuasion Through Emotion? An Experimental Test of the Emotion-Eliciting Nature of Populist Communication. In: International Journal of Communication 12, 1114-1138.

Žižek, Slavoj (2001): Die Tücke des Subjekts. Frankfurt am Main: Suhrkamp. 
